\title{
Activating and inhibitory IgG Fc receptors on human DCs mediate opposing functions
}

\author{
Adam M. Boruchov, 1,2,3,4,5 Glenn Heller,4,6 Maria-Concetta Veri,7 Ezio Bonvini,7 \\ Jeffrey V. Ravetch, ${ }^{8}$ and James W. Young $1,3,4,5,9$
}

\begin{abstract}
1'Laboratory of Cellular Immunobiology, ${ }^{2}$ Hematology Service, Division of Hematologic Oncology, ${ }^{3}$ Department of Medicine, ${ }^{4}$ Memorial Sloan-Kettering Cancer Center, New York, New York, USA. ${ }^{5}$ Weill Medical College of Cornell University, New York, New York, USA. ${ }^{6}$ Biostatistics Service, Department of Biostatistics and Epidemiology, Memorial Sloan-Kettering Cancer Center, New York, New York, USA. ${ }^{7}$ MacroGenics, Rockville, Maryland, USA. ${ }^{8}$ Leonard Wagner Laboratory of Molecular Genetics and Immunology, The Rockefeller University, New York, New York, USA. ${ }^{9}$ Allogeneic Bone Marrow Transplantation and Clinical Immunology Services, Division of Hematologic Oncology, Department of Medicine, Memorial Sloan-Kettering Cancer Center, New York, New York, USA.
\end{abstract}

\begin{abstract}
Human monocyte-derived DCs (moDCs) and circulating conventional DCs coexpress activating (CD32a) and inhibitory (CD32b) isoforms of IgG Fc $\gamma$ receptor $(\mathrm{Fc} \gamma \mathrm{R}) \mathrm{II}(\mathrm{CD} 32)$. The balance between these divergent receptors establishes a threshold of DC activation and enables immune complexes to mediate opposing effects on DC maturation and function. IFN- $\gamma$ most potently favors CD32a expression on immature DCs, whereas soluble antiinflammatory concentrations of monomeric IgG have the opposite effect. Ligation of CD32a leads to DC maturation, increased stimulation of allogeneic $T$ cells, and enhanced secretion of inflammatory cytokines, with the exception of IL-12p70. Coligation of CD32b limits activation through CD32a and hence reduces the immunogenicity of moDCs even for a strong stimulus like alloantigen. Targeting CD32b alone does not mature or activate DCs but rather maintains an immature state. Coexpression of activating and inhibitory Fc $\gamma \mathrm{Rs}$ by DCs reveals a homeostatic checkpoint for inducing tolerance or immunity by immune complexes. These findings have important implications for understanding the pathophysiology of immune complex diseases and for optimizing the efficacy of therapeutic $m A b s$. The data also suggest novel strategies for targeting antigens to the activating or inhibitory $F c \gamma$ Rs on human DCs to generate either antigen-specific immunity or tolerance.
\end{abstract}

\section{Introduction}

mAbs are among the most rapidly growing therapies for the treatment of cancer (1) and autoimmunity (2). Antibodies either fix complement or engage cells of the innate immune system to mediate target cell lysis. The latter process, known as antibody-dependent cellular cytotoxicity (ADCC), requires that the Fc portion

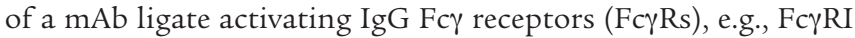
(CD64), Fc $\gamma$ RIIa (CD32a), Fc $\gamma$ RIIc (CD32c), or Fc $\gamma$ RIII (CD16), on monocytes, NK cells, neutrophils, or DCs (3). Recent evidence suggests a more indirect effector mechanism, in which Fc $\gamma$ Rs on DCs mediate phagocytosis and enhance cross-presentation of antibodycoated antigens, leading to effective stimulation of both $\mathrm{CD}^{+} \mathrm{Th} 1$ and $\mathrm{CD}^{+} \mathrm{CTL}$ effector responses (4-7). Studies in mice show that coligation of the unique inhibitory Fc $\gamma \mathrm{RIIb}(\mathrm{CD} 32 \mathrm{~b})$ abrogates all of these effects $(7,8)$.

The activating and inhibitory FcyRs on DCs offer rational targets for immunotherapy based on the unique capacity of DCs to play critical roles in both immunity and tolerance (9). Studies in mice have been very promising (7), though translation into the human system has been lacking. Investigators have not been able to distinguish surface CD32a and CD32b when coexpressed on human cells, given their highly homologous extracellular domains (3). In addition, a common genetic polymorphism of CD32a

Nonstandard abbreviations used: ADCC, antibody-dependent cellular cytotoxicity; alloMLR, allogeneic mixed leukocyte reaction; $\mathrm{Fc} \gamma \mathrm{R}, \mathrm{Fc} \gamma$ receptor; $\left[{ }^{3} \mathrm{H}\right] \mathrm{TdR}$, methyl$\left[{ }^{3} \mathrm{H}\right]$ thymidine; IVIG, intravenous Ig; MFI, mean fluorescence intensity; moDC, monocyte-derived DC; NHS, normal human serum.

Conflict of interest: J.V. Ravetch has a financial interest in MacroGenics Inc., which is managed by Rockefeller University's conflict of interest committee.

Citation for this article: J. Clin. Invest. 115:2914-2923 (2005).

doi:10.1172/JCI24772. caused by an arginine $(\mathrm{R})$ to histidine $(\mathrm{H})$ amino acid substitution at position 131 yields divergent avidities for mouse and human IgG ligands (10), which further confounds studies of Fc $\gamma \mathrm{R}$ function in the human system.

We have used a recently developed $\mathrm{mAb}$ that, unlike any other available reagent, can specifically bind the inhibitory $\mathrm{CD} 32 \mathrm{~b}$ isoform, as well as block its interaction with IgG, on intact human cells (M.C. Veri et al., unpublished observations). We have evaluated the relative expression of the activating CD16, CD32a, and CD64, in addition to the inhibitory CD32b, on circulating DCs and their precursors as well as on cytokine-induced monocytederived DCs (moDCs). We have demonstrated the phenotypic and functional sequelae of ligating either or both the activating CD32a and inhibitory CD32b on immature moDCs. We have also identified factors that modulate the balanced expression of these receptors, which in turn affect the IgG-mediated changes in maturation and function of the DCs themselves. Our findings have important implications for understanding the pathophysiology of diseases mediated by immune complexes and for developing and optimizing antibody- and DC-based therapies for antigen-specific immunity or tolerance. The data also suggest the need for further studies to define the cell biology of enhanced processing and presentation conferred by antigen opsonization.

\section{Results}

Specific mAbs identify CD32 isoforms and CD32a allelic variants by flow cytometry. We first validated the specificity of mAbs for this study using neutrophils and B cells that express only CD32a or CD32b, respectively, on the cell surface. The novel clone $2 \mathrm{~B} 6$, which binds extracellular CD32b exclusive of CD32a (M.C. Veri et al., unpublished observations), stained B cells but not neutrophils (Fig- 


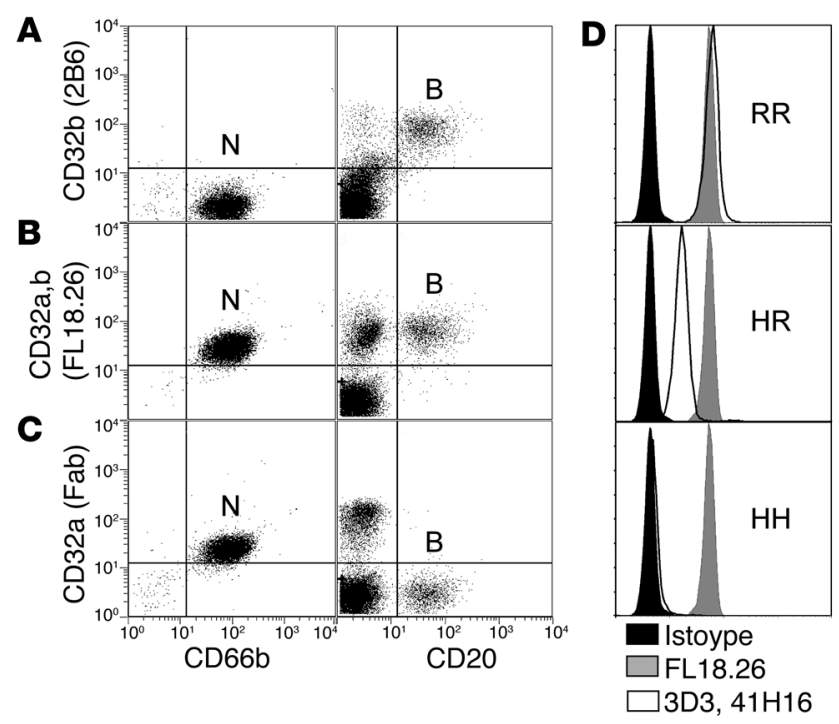

ure 1A). Clone FL18.26 is not isoform specific (11) and stained neutrophils and B cells (Figure 1B). In contrast, Fab fragments of IV.3 are CD32a specific $(12,13)$ and detected neutrophils but not B cells (Figure 1C). These data confirm the specificity of $2 \mathrm{~B} 6$ for CD32b and Fabs of IV.3 for CD32a, thus enabling a clear distinction between activating and inhibitory isoforms of CD32 expressed on the cell surface.

The $\mathrm{R}$ and $\mathrm{H}$ variants of CD32a have differing avidities for mouse and human IgG subtypes. Compared with the CD32 $\mathrm{a}_{131} \mathrm{R}$ allotype, CD32 $\mathrm{a}_{131} \mathrm{H}$ has a higher avidity for complexed human IgG2 and IgG3 (14) and, to a lesser extent, human IgG1 (15). Unlike CD $32 \mathrm{a}_{131} \mathrm{H}$, however, CD32 $\mathrm{a}_{131} \mathrm{R}$ binds complexed mouse IgG1 (10). We therefore determined the CD32a phenotypes of all samples used in functional studies, based on staining of neutrophils that express abundant CD32a only. mAbs 3D3 and 41H16 recognize only the $R_{131}$ variant of CD32a (16), whereas mAbs FL18.26 and IV.3 recognize both the $R_{131}$ and $H_{131}$ variants (11, 14). As shown in Figure 1D, FL18.26 stained neutrophils from all CD32a phenotypes with equal intensity. In contrast, 3D3 stained cells homozygous for CD32 $\mathrm{a}_{131} \mathrm{R}$, did not detect cells homozygous for $\mathrm{CD} 32 \mathrm{a}_{131} \mathrm{H}$, and displayed intermediate staining of heterozygous samples. We confirmed that this method accurately typed U937, THP-1, and K562 cell lines expressing known CD32a subtypes $(17,18)$ (data not shown). These data

\section{Figure 2}

Monocytes, circulating conventional DCs, and cytokine-induced moDCs all express a range of $\mathrm{Fc} \gamma \mathrm{Rs}$, whereas freshly isolated plasmacytoid DCs lack detectable surface expression of all Fc $\gamma$ Rs. Freshly isolated PBMCs were labeled with fluorochrome-conjugated mAbs. (A) After gating on HLA-DR bright PBMCs that were lineage marker negative, CD32a (left) and CD32b (right) were detected on CD123low conventional DCs (conv. DCs) but not on CD123 $3^{\text {bright }}$ plasmacytoid DCs (pDCs). (B) Monocytes were identified as CD14+ PBMCs. moDCs were studied as immature cells, gated according to characteristic phenotype (48) but lacking the surface CD83 expression of mature moDCs. Open histograms correspond to isotype controls, and filled histograms represent staining of the indicated $\mathrm{Fc} \gamma \mathrm{R}$. Most often, CD32a and CD32b were coexpressed on the same subpopulation of moDCs, as shown by a representative sample in $\mathbf{C}$.

\section{Figure 1}

$2 \mathrm{~B} 6$ is a novel mAb that specifically detects an extracellular domain of CD32b. Neutrophils and PBMCs were isolated from peripheral blood samples. Cells were stained with various anti-CD32 mAbs and counterstained with anti-CD66b to define neutrophils (N) or anti-CD20 to define $B$ cells (B). (A) mAb 2B6 detected CD32b on B cells but not CD32a on neutrophils. (B) mAb FL18.26 detected CD32a or CD32b, and it stained neutrophils as well as B cells. (C) In contrast, mAb IV.3 (Fab) detected CD32a on neutrophils but not CD32b on B cells. (D) Some mAbs were able to distinguish between the common polymorphic variants of CD32a. mAbs FL18.26 (B) and IV.3 (C) bind both the $\mathrm{R}_{131}$ and $\mathrm{H}_{131}$ subtypes of CD32a and stain neutrophils from $\mathrm{HH}$ homozygotes, RR homozygotes, and HR heterozygotes equally. mAbs 3D3 and $41 \mathrm{H} 16$ recognize only the $\mathrm{R}_{131}$ subtype (16), shown staining neutrophils from CD32 $\mathrm{a}_{131} \mathrm{RR}$ individuals but not from CD32 $\mathrm{a}_{131} \mathrm{HH}$ individuals, with intermediate staining of neutrophils from heterozygous $\left(\mathrm{CD} 32 \mathrm{a}_{131} \mathrm{HR}[\mathrm{HR}]\right)$ individuals.

validate this quick method for distinguishing the polymorphic phenotypes of CD32a by flow cytometry (14).

Subtypes of freshly isolated $D C s$ and $D C$ precursors have distinct $F c \gamma R$ expression profiles. We studied the differential expression of the activating and inhibitory Fc $\gamma$ Rs on freshly isolated populations of DCs and their precursors in peripheral blood. We identified "myeloid" or conventional DCs from freshly isolated PBMCs as being lineage $\left(\mathrm{CD} 3, \mathrm{CD} 14, \mathrm{CD} 20\right.$, and CD56) ${ }^{\text {negative }}$, HLA-DR ${ }^{\text {bright }}, \mathrm{CD} 11 \mathrm{c}^{+}$, and CD123 low, whereas plasmacytoid DC precursors were lineagenegative, HLA-DR ${ }^{\text {bright }}$, CD11 $\mathrm{c}^{\text {negative }}$, and CD123 $3^{\text {bright }}$ (19).

Consistent with previously published results, we found that $\mathrm{CD} 11 \mathrm{c}^{+} \mathrm{CD} 123^{\text {low }}$ conventional DCs in peripheral blood expressed the activating FcyRs CD32a and CD64 but did not express CD16 (4). Almost all circulating conventional DCs also expressed the inhibitory CD32b $(n=10$; mean $\pm \mathrm{SD}, 92 \% \pm 3.3 \%)$ (Figure $2 \mathrm{~A})$. We confirmed that freshly isolated plasmacytoid DCs, however, did not express detectable surface levels of CD16, CD32b, or CD64 and expressed minimal to no CD32a (Figure 2A) (20). All CD14 ${ }^{+}$ monocytes, which are circulating precursors of moDCs, expressed
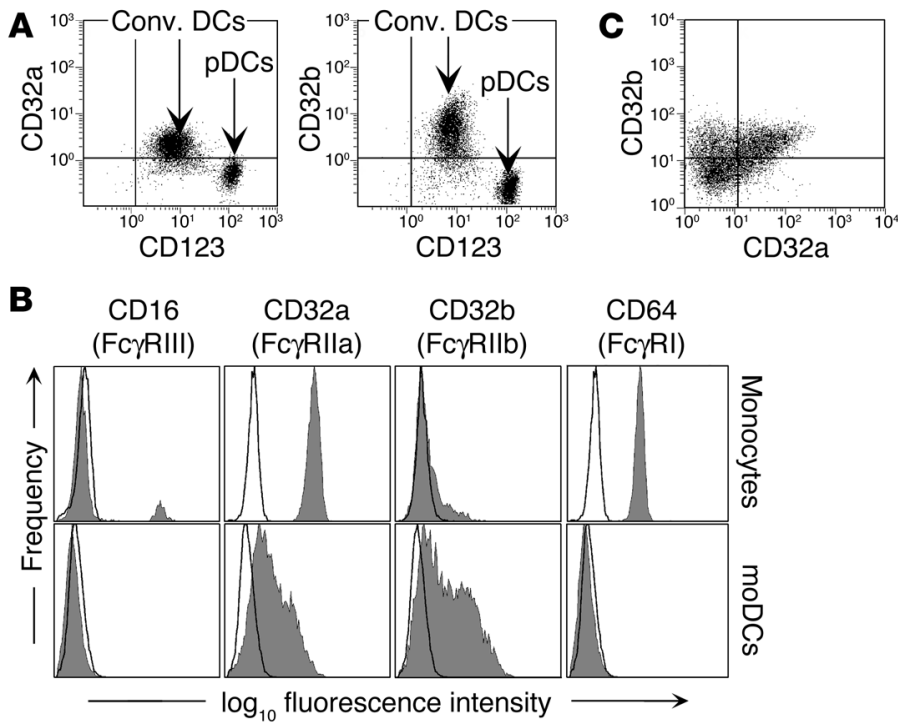
CD32a and CD64 (Figure 2B). Monocyte coexpression of the inhibitory CD32b was highly variable among 30 healthy volunteers, however, ranging from $1 \%$ to $48 \%$ (mean, $18.1 \%$, SD $=8.3$ ). A separate, but partially overlapping, small subpopulation of CD14 low monocytes expressed CD16 (Figure 2B) $(21,22)$. Immature moDCs expressed a balance of CD32a and CD32b similar to that of conventional DCs circulating in fresh blood. On average, $56 \% \pm 10.7 \%$ of immature moDCs expressed CD32a $(n=17)$ and $64 \% \pm 9.4 \%$ expressed CD32b ( $n=17$ ) (Figure 2B). Most often, these divergent receptors were coexpressed on the same population of cells (Figure 2C). Unlike their monocyte precursors, moDCs lost expression of CD16 and CD64 by day 1-2 of differentiation. Immature moDCs thus provide an excellent model for studying the modulation and function of CD32a and CD32b on the same cells.

Various stimuli modulate the balanced expression of CD32 $a$ and $C D 32 b$ on immature moDCs. We tested the effects of several immune modulators on the balance of activating and inhibitory Fc $\gamma$ Rs on immature moDCs. Figure 3 shows mean fold changes in the percentage of cells expressing the respective Fc $\gamma$ Rs (Figure 3A) and the relative changes in $\mathrm{Fc} \gamma \mathrm{R}$ density on the cell surface (Figure $3 \mathrm{~B}$ ) compared with untreated moDCs from the same donors ( $n=6$ independent experiments). Fc $\gamma \mathrm{R}$ density was measured as the number of anti-Fc $\gamma$ R detection mAbs bound per cell. Mean fluorescence intensities (MFIs) and shifts in MFIs were identical for $\mathrm{F}\left(\mathrm{ab}^{\prime}\right)_{2}$ and whole IgG 2B6 (anti-CD32b), which indicates that Fc $\gamma$ R staining was mediated by Fab-specific binding and not by interactions with the Fc portions of the detection mAbs. Upregulation of $\mathrm{Fc} \gamma \mathrm{Rs}$ by the agents tested did not increase nonspecific Fc-mediated binding (Figure 3C).

All factors affected only CD32a and/or CD32b expression, or neither, except IL-10 and IFN- $\gamma$, which also induced expression of CD16 and CD64, respectively (data not shown). IL-10, IL-6, and dexamethasone all led to proportional increases in expression of CD32a and CD32b without a clear shift favoring either isoform. IFN- $\gamma$ most potently shifted the balance in favor of activating $\mathrm{Fc} \gamma \mathrm{Rs}$ by inducing expression of CD64, increasing the frequency and density of CD32a expression, and exerting opposing effects on CD32b. Conversely, antiinflammatory concentrations of soluble monomeric $\operatorname{IgG}(0.15 \mathrm{mM})$, approximating the levels achieved in vivo after administration of intravenous Ig (IVIG), decreased CD32a expression and yielded little to no increase in CD32b expression. Among all factors tested, this led to the greatest relative shift in favor of the

\section{Figure 3}

Various stimuli modulate the balanced expression of CD32a and CD32b on immature moDCs. The indicated reagents were added to cultures of immature moDCs from day 3 to day 6 . Expression of Fc $\gamma$ Rs was measured by flow cytometry (CD16 and CD64 not shown). Analyzed cells were immature or specifically gated for the absence of CD83 in cultures where there was a small amount of maturation $\left(\mathrm{PGE}_{2}\right.$ and TNF- $\alpha$ ). The mean fold changes ( $\pm \mathrm{SD}$ ) in the frequency of cells expressing a given $\mathrm{F} c \gamma \mathrm{R}$ induced by each reagent, compared with untreated cells, are shown in A. Density was calculated on the $\mathrm{Fc}_{\mathrm{C}} \mathrm{R}^{+}$cells as the number of anti-Fc $\gamma \mathrm{R}$ antibodies bound per cell using a commercially available kit. The mean fold changes $( \pm S D)$ in $F c \gamma R$ density induced by the reagents in 5 independent experiments, compared with the averaged $\mathrm{Fc \gamma R}$ densities on untreated/control moDCs, are shown in B. Sample histograms for untreated immature moDCs and IFN- $\gamma$-treated immature moDCs are shown in C. Open histograms correspond to isotype controls, and filled histograms show staining by the indicated anti-FcyR mAbs. inhibitory Fc $\gamma$ Rs. CD32a and CD32b did not bind soluble monomeric IgG, which was not detected on the surface of moDCs using anti-human IgG antibodies (data not shown). Hence, receptor occupancy could not account for any change in detection of CD32 isoforms. Culturing cells in the presence of $10 \%$ FCS or TNF- $\alpha$ potently reduced the frequency and density of $\mathrm{Fc} \gamma \mathrm{R}$ expression. All tested maturation stimuli decreased the frequency of CD32a- and CD32b-expressing cells. However, LPS and CD40L, but not the combination of IL- $1 \beta$, IL- 6 , TNF- $\alpha$, and PGE 2 (23), shifted the balance of remaining Fc $\gamma$ R-expressing cells in favor of CD32a.

We tested the effects of factors on circulating conventional and plasmacytoid DCs enriched from whole blood after negative selection and cultured in Teflon beakers in 10\% normal human

A

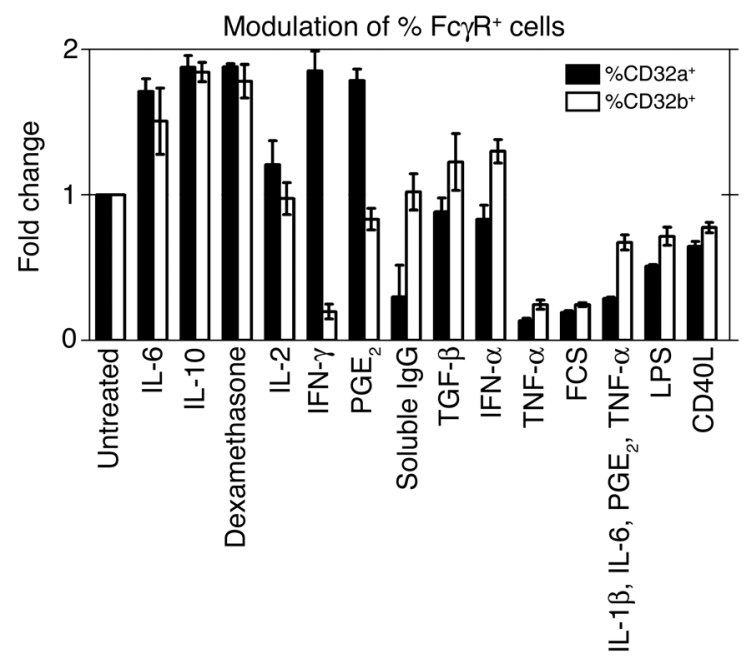

B
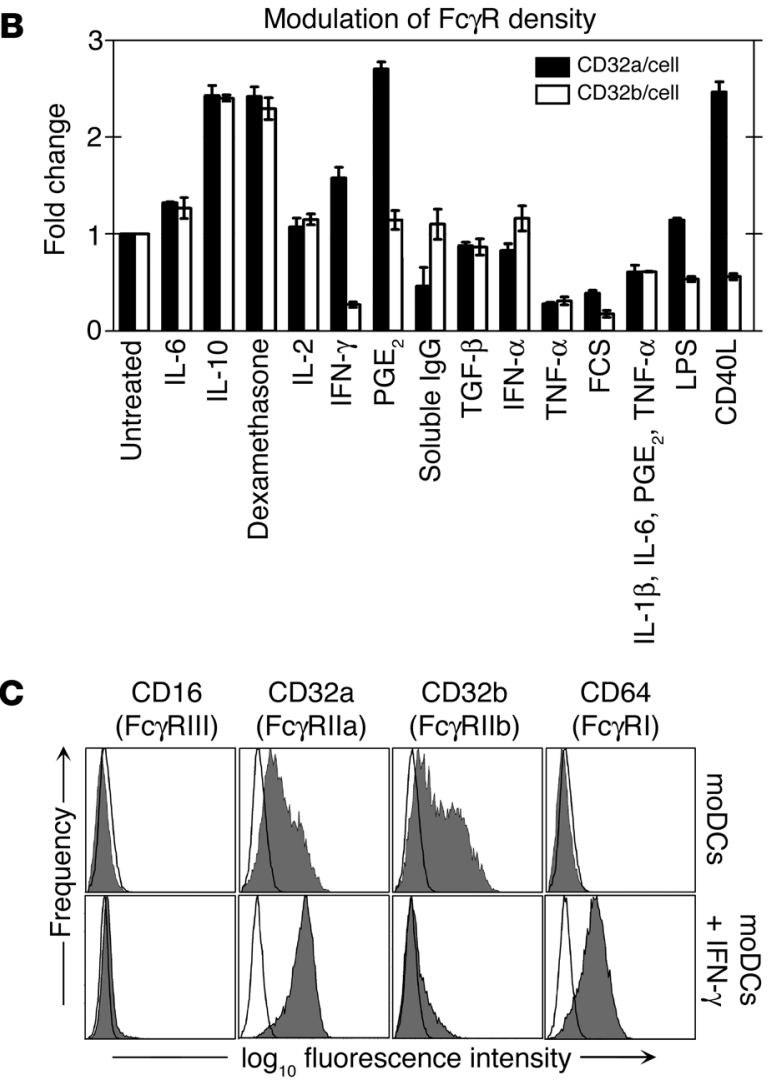


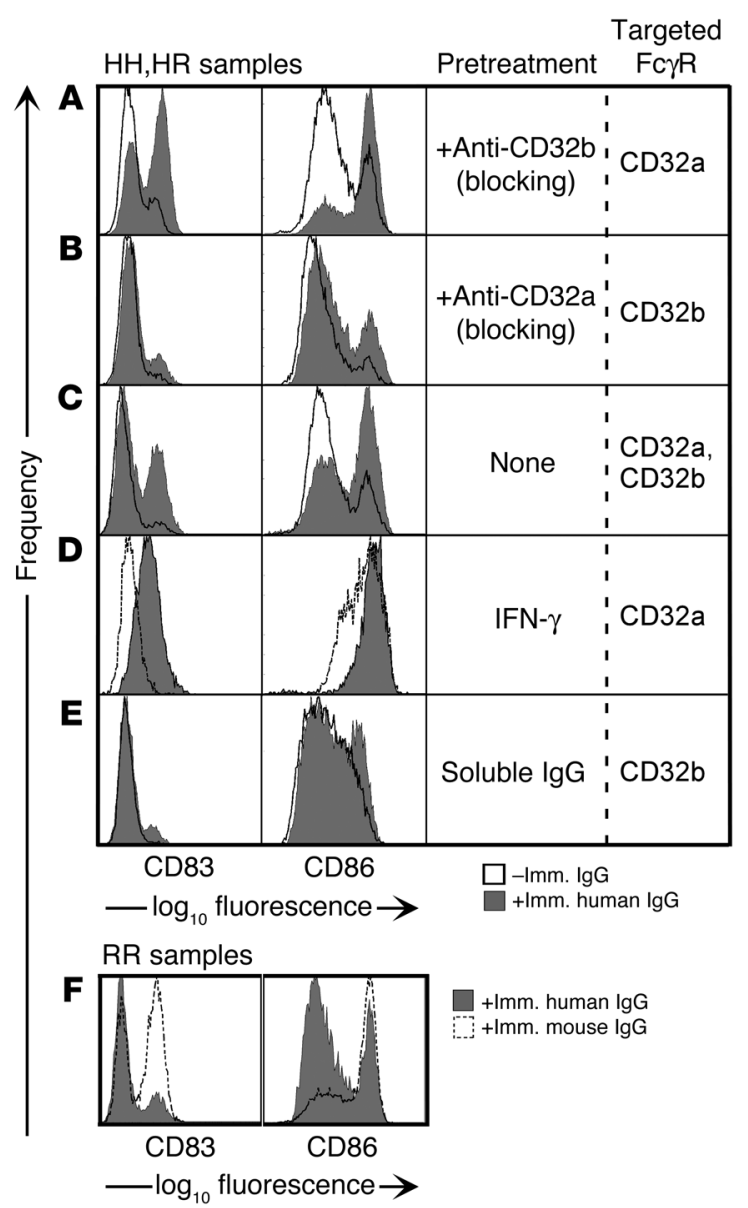

serum-RPMI (NHS-RPMI). We added IL-3 to support the viability of plasmacytoid DCs in culture (24). IFN- $\alpha$, IFN- $\gamma$, TGF- $\beta$, IL-10, and soluble IgG modulated CD32a and/or CD32b on circulating conventional DCs, which was similar to their effects on cytokineinduced moDCs. Unlike freshly isolated cells, plasmacytoid DCs in culture expressed CD32a, which was modulated by these factors in the same manner as on moDCs (data not shown).

$C D 32 a$ and CD32b have opposing effects on DC maturation. We studied the effects of ligating human IgG to CD32a, CD32b, or both

\section{Figure 4}

Ligation of CD32a or CD32b on immature moDCs has opposing effects on maturation phenotype. (A-E) MoDCs were cultured on plates with immobilized (Imm.) human IgG to ligate Fc $\gamma$ Rs (filled histograms). CD32a (A) or CD32b (B) was specifically ligated by first incubating moDCs with blocking antibodies against either CD32b or CD32a, respectively. CD32a and $\mathrm{CD} 32 \mathrm{~b}$ were ligated simultaneously $(\mathbf{C})$ by preincubating moDCs without blocking antibodies. DCs with or without blocking antibodies were also cultured on untreated plates as negative controls (open histograms). Cells were harvested at 48 hours, and DC phenotype was assessed by flow cytometry. Histograms from 1 representative experiment of 8 that used $\mathrm{CD} 32 \mathrm{a}_{131} \mathrm{HH}$ or $-\mathrm{HR}$ samples are shown in A-C. Immature IFN- $\gamma$-treated moDCs (D) and soluble IgGtreated moDCs (E) were washed to remove these factors and recultured with (filled histograms) or without (open histograms) immobilized human IgG. Cells were harvested at 24-48 hours and phenotype was assessed by flow cytometry. Representative histograms from 1 of 5 separate experiments are shown. (F) In contrast to results obtained from $\mathrm{CD} 32 \mathrm{a}_{131} \mathrm{HH}$ or $-\mathrm{HR}$ samples (C, filled histograms), CD32 $\mathrm{a}_{131} \mathrm{RR}$ samples were not matured to the same extent after coculture with immobilized human IgG (F, filled histograms; $n=4$ experiments). Immobilized mouse IgG1 (F, open histograms), which ligates CD32a but not CD32b in CD32a $a_{131} R R$ individuals (10), led to maturation that was similar to conditions specifically targeting CD32a on $\mathrm{CD} 32 \mathrm{a}_{131} \mathrm{HH}$ or -HR samples (A, filled histograms). Averaged changes in CD83 and CD86 expression are summarized in Table 1.

on immature moDCs, using immobilized IgG to mimic complexed IgG (25). Selective ligation of CD32a matured a subpopulation of moDCs, as evidenced by upregulation of the DC maturation marker CD83 (26) and the costimulatory molecule CD86 (average $58 \% \mathrm{CD}^{\circ} 3^{+}$and $79 \% \mathrm{CD}^{+} ; n=8$ experiments; Figure $4 \mathrm{~A}$, filled histograms). The frequency of maturation was proportional to the percentage of cells that expressed CD32a. Blocking CD32b in the absence of a ligand for CD32a (recultured in complete medium with $1 \%$ NHS, GM-CSF, and IL-4 but without immobilized IgG) did not promote DC maturation (9\% CD83 ${ }^{+}$; Figure $4 \mathrm{~A}$, open histograms) compared with untreated controls $(8 \%$ $\mathrm{CD}^{+} 3^{+}$; Figure 4C, open histograms) in a total of 8 independent experiments. Ligation of $\mathrm{CD} 32 \mathrm{~b}$ was associated with a minimal increase in maturation (average 10\% increase in CD83; Figure 4B). When the $2 \mathrm{Fc} \gamma \mathrm{Rs}$ were targeted simultaneously, CD32b limited

Table 1

Average changes in total moDCs expressing CD83 and CD86

\begin{tabular}{|c|c|c|c|c|c|}
\hline \multirow[t]{2}{*}{ CD32a allotype } & \multirow[t]{2}{*}{ Pretreatment } & \multirow[t]{2}{*}{$\begin{array}{c}\text { Fc } \gamma R \text { ligand } \\
\text { (immobilized IgG) }\end{array}$} & \multirow[t]{2}{*}{ Targeted $\mathbf{F} \mathbf{c} \gamma \mathbf{R}$} & \multicolumn{2}{|c|}{$\begin{array}{l}\text { Average increase in percen } \\
\text { of total moDCs expressing }\end{array}$} \\
\hline & & & & CD83 & CD86 \\
\hline $\mathrm{CD}_{2} 2 \mathrm{a}_{131} \mathrm{HR}$ & Anti-CD32b & Pooled human IgG & CD32a & 49 & 41 \\
\hline \multirow[t]{4}{*}{ CD32a $\mathrm{a}_{131} \mathrm{HH}$ samples } & Anti-CD32a & Pooled human IgG & CD32b & 10 & 12 \\
\hline & None (medium) & Pooled human IgG & CD32a, CD32b & 27 & 31 \\
\hline & IFN- $\gamma$ & Pooled human IgG & CD32a & 49 & 24 \\
\hline & $\operatorname{slg} G$ & Pooled human IgG & CD32b & 6 & 7 \\
\hline \multirow[t]{2}{*}{ CD32a RR samples } & None & Pooled human IgG & CD32a, CD32b & 7 & 13 \\
\hline & None & Mouse $\lg G 1$ & $\mathrm{CD} 32 \mathrm{a}>\mathrm{CD} 32 \mathrm{~b}$ & 45 & 47 \\
\hline
\end{tabular}

FC $\gamma$ Rs on moDCs were ligated by immobilized IgG after pretreatment with blocking mAbs (anti-CD32a, anti-CD32b), IFN- $\gamma$, soluble IgG (slgG), or medium control. Data in the 2 far right columns represent the average increases in the percent of total moDCs expressing the indicated epitope over those not exposed to immobilized IgG. 
CD32a-induced maturation (Figure 4, C vs. A, filled histograms; $P=0.001)$. Table 1 summarizes the average increases in the percent of total moDCs expressing CD83 and CD86 after coculture with immobilized IgG, compared with that of similarly treated moDCs cultured without immobilized IgG.

Cytokine- or soluble IgG-induced shifts in the balance between activating and inbibitory Fc $\gamma$ Rs affect susceptibility to immobilized IgG-mediated maturation. We pretreated moDCs with factors that modulated the balance in favor of either activating or inhibitory FcyRs, washed the cells to remove these factors, then recultured the moDCs in complete medium with $1 \%$ NHS, GM-CSF, and IL-4, in the presence or absence of immobilized IgG. Culturing IFN- $\gamma$-treated DCs in the presence of immobilized IgG led to increased DC maturation compared with cultures lacking immobilized IgG (62\% CD83+ vs. $13 \% \mathrm{CD}^{2} 3^{+}$in 4 experiments; Figure 4D). The relative increase in maturation was similar to conditions in which CD32b was blocked before coculture with immobilized IgG (Figure 4A). Conversely, culturing soluble IgG-treated moDCs with immobilized IgG led to only a 6\% increase in CD83 expression (Figure $4 \mathrm{E}$ ). These data indicated that immobilized IgG ligated predominantly activating Fc $\gamma$ Rs on IFN- $\gamma$-treated moDCs or CD32b on IgG-treated moDCs. Pharmacologic modulations of the balance between CD32a and CD32b could therefore affect IgG-mediated maturation.

Differences in affinity for IgG ligand cause a functional shift in the balance between CD32a and CD32b. Coculturing untreated moDCs from CD $32 \mathrm{a}_{131} \mathrm{HH}$ or CD $32 \mathrm{a}_{131} \mathrm{HR}$ individuals with immobilized IgG (Figure 4C, filled histogram) yielded a greater increase in maturation compared with moDCs from RR individuals (average absolute increase in $\mathrm{CD}^{2} 3^{+}$cells: $27 \%$ vs. $7 \%$; filled histograms in Figure 4, C and F, respectively; $P<0.001$ ). Immobilized mouse IgG1 in the same RR samples, however, led to substantial maturation (Figure 4F, open histograms) and a loss of the discrepancy between the different CD32 $\mathrm{a}_{131}$ phenotypes. In the context of a fixed quantity of Fc $\gamma$ Rs, differences in ligand avidity can thus cause a shift in the functional balance between CD32a and CD32b.

Coligation of $C D 32 b$ limits $C D 32 a$-mediated cytokine release. After ligating IgG to CD32a, CD32b, or both, as outlined above, we collected cell-free supernatants at 24-48 hours and measured a panel of cytokines using a multiplexed bead assay. Samples

\section{Figure 5}

Coligation of CD32b limits CD32a-mediated cytokine release. Immature moDCs were cocultured with immobilized IgG to target Fc $\gamma$ Rs. After 2 days, supernatants were collected and cytokines measured using a flow cytometry-based multiplexed bead assay. Mean cytokine levels (picograms per milliliter) are plotted on the $y$ axis. (A) For samples derived from $\mathrm{CD} 32 \mathrm{a}_{131} \mathrm{HH}$ or -HR donors, CD32a, CD32b, or both were ligated on immature moDCs after first blocking or not with the mAb to the other isoform ( $n=6$ independent experiments). $P$ values for each cytokine reflect differences between CD32a-targeted DCs and DCs on which CD32a and $\mathrm{CD} 32 \mathrm{~b}$ were targeted simultaneously. (B) IFN- $\gamma-$ and soluble IgG-mediated shifts in the expression of CD32a and CD32b were also assessed ( $n=4$ independent experiments). $P$ values for IFN- $\gamma$-treated DCs (top) and IgG-treated DCs (bottom) reflect differences between DCs from respective conditions cocultured with or without immobilized IgG. (C) Immobilized mouse IgG was used as the ligand for Fc $\gamma$ Rs from CD32 $a_{131}$ RR samples $(n=3$ independent experiments). from $\mathrm{CD} 32 \mathrm{a}_{131} \mathrm{HH}$ and -HR individuals are shown in Figure 5A. Simultaneous ligation of CD32b and CD32a (no pretreatment with blocking mAbs) led to suppressed levels of inflammatory cytokines compared with targeting CD32a alone. Differences were statistically significant for TNF- $\alpha(P<0.001)$, IL-6 $(P=0.02)$, and IL-8 $(P=0.002)$ secretion $(n=6$ experiments). Significant levels of IL- $1 \beta$ and IL-12p70 were not detected under any condition, and therefore results are not shown. These data offer further functional evidence of the inhibitory role of CD32b.

We also tested cytokine release after ligation of immobilized human IgG to Fc $\gamma$ Rs on IFN- $\gamma$ - and soluble IgG-treated immature moDCs (CD32 $\mathrm{a}_{131} \mathrm{HH}$ and -HR samples), which favor the respective expression of either CD32a or CD32b. Soluble IgG-treated moDCs cocultured with immobilized IgG did not increase secretion of TNF- $\alpha$, IL-6, or IL-8, compared with soluble IgG-treated

A
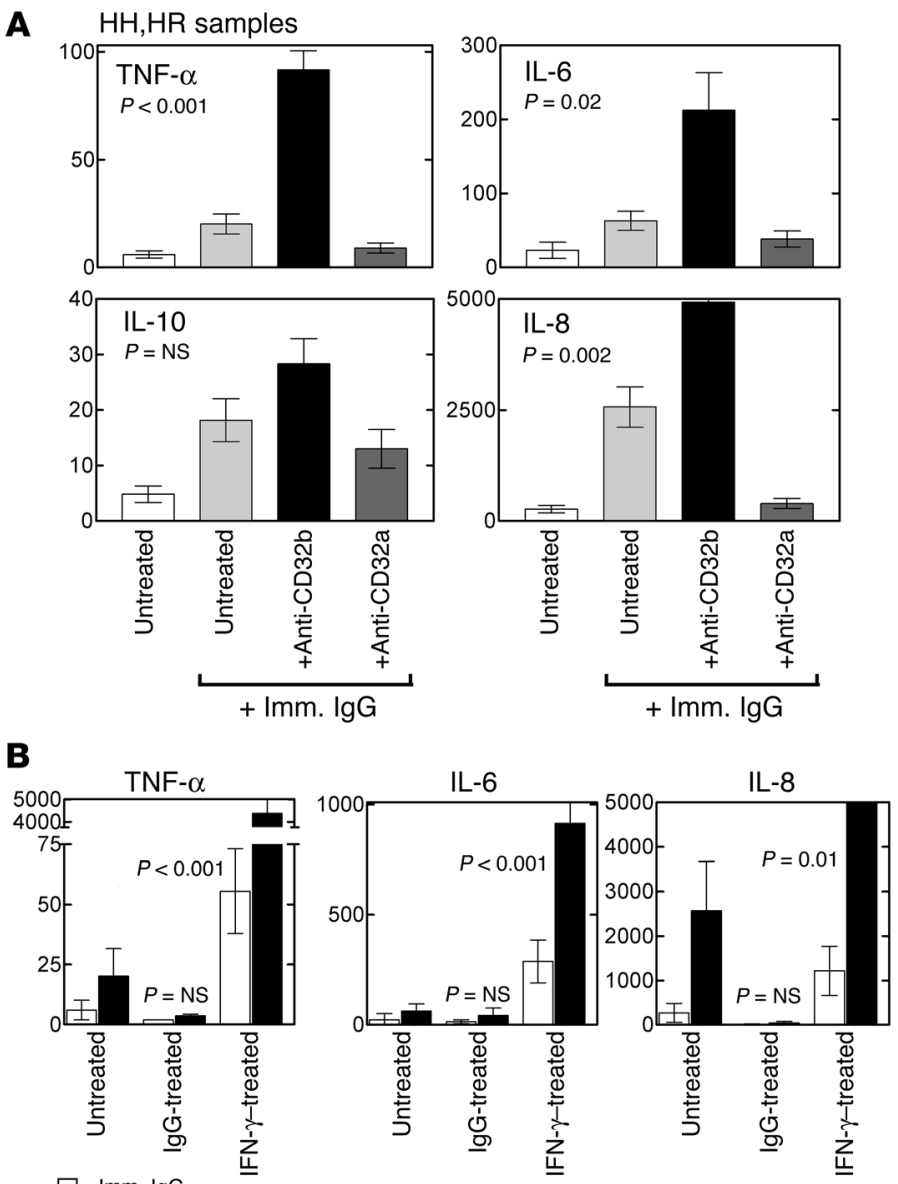

$-\mathrm{mm} . \operatorname{lgG}$
$+\mathrm{Imm} . \operatorname{lgG}$

C
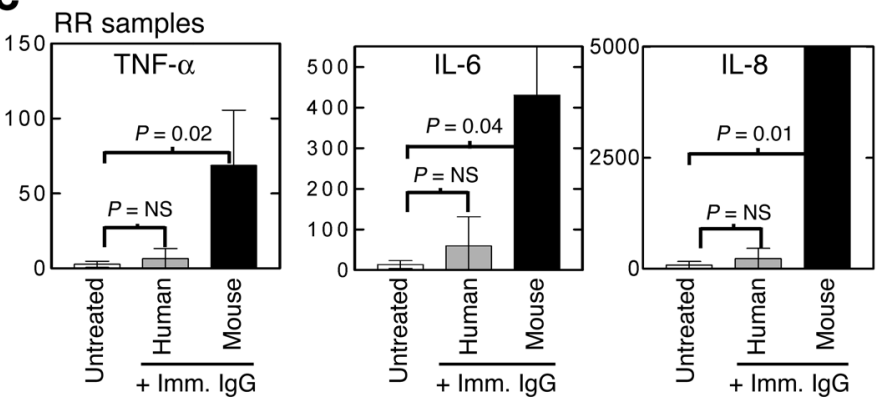

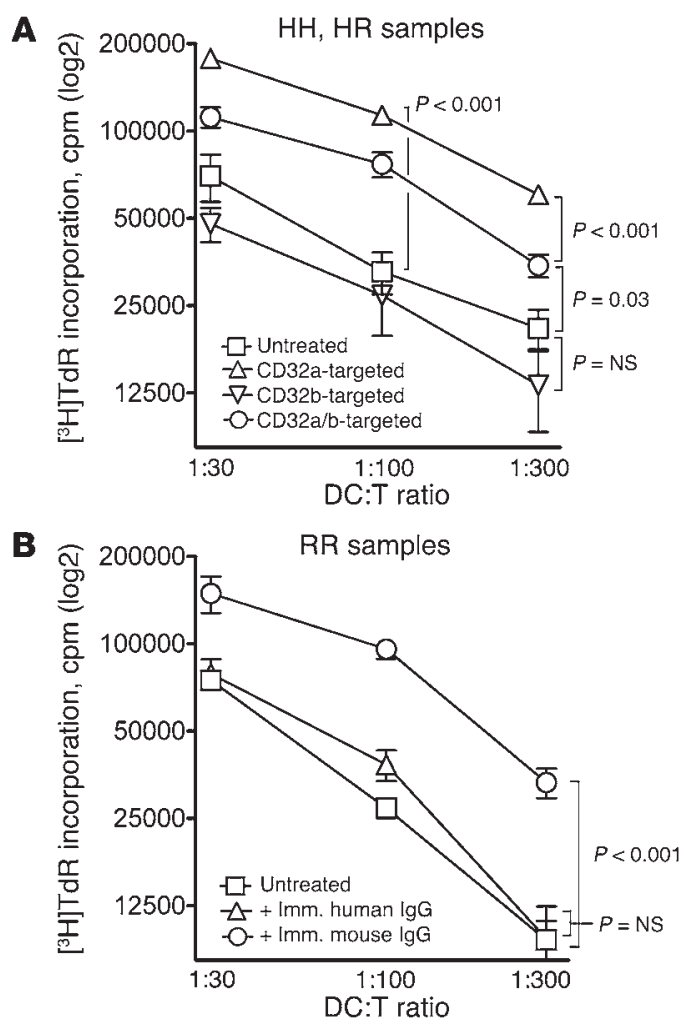

moDC controls (Figure 5B). In contrast, ligating Fc $\gamma$ Rs on IFN- $\gamma$ treated DCs led to enhanced secretion of TNF- $\alpha(P<0.001)$, IL-6 $(P<0.001)$, and IL-8 $(P=0.01)$ compared with IFN- $\gamma$-treated DCs cultured without immobilized IgG (Figure 5B). The results were similar to those for DCs on which CD32a alone was ligated (Figure 5A).

The $H H / H R$ versus $R R$ phenotypes have functional significance in cytokine release assays. Compared with samples from CD $32 \mathrm{a}_{131} \mathrm{HH}$ and -HR individuals (Figure 5A), coculturing moDCs from CD32 $\mathrm{a}_{131} \mathrm{RR}$ individuals with immobilized human IgG did not significantly enhance cytokine production (Figure 5C). Because the CD32 $\mathrm{a}_{131} \mathrm{R}$ subtype has a high affinity for murine IgG1 (10), however, immobilized mouse IgG (Figure 5C) led to marked increases in TNF- $\alpha(P=0.02)$, IL-6 $(P=0.04)$, and IL-8 $(P=0.01)$ levels that were similar to those in CD32a-ligated DCs from CD32 $\mathrm{a}_{131} \mathrm{HH}$ or -HR individuals (Figure 5A).

Taken together, the results indicate that shifts in the expression of CD32a and CD32b have functional consequences for moDC cytokine secretion. CD32a variants also determine moDC susceptibility to human or mouse Ig and hence the efficacy of certain $\mathrm{mAb}$ therapies (27).

Targeting CD32a versus $C D 32 b$ has opposing effects on DC stimulatory capacity in the allogeneic mixed leukocyte reaction. We targeted CD32a, CD32b, both, or neither, on immature moDCs and measured effects on DC immunogenicity using allogeneic mixed leukocyte reactions (alloMLRs). Results from 5 experiments using $\mathrm{CD} 32 \mathrm{a}_{131} \mathrm{HH}$ and -HR samples are shown in Figure 6A. CD32atargeted DCs were the most potent stimulators of allogeneic $\mathrm{T}$ cells $(P<0.001$; Figure 6 A, triangles) because of the maturation effect of ligating CD32a. Coligation of CD32b (circles) limited the absolute increase in stimulatory capacity otherwise medi-

\section{Figure 6}

Targeting CD32a or CD32b affects DC allostimulatory capacity in the MLR. Two days after ligating CD32a, CD32b, both, or neither on immature moDCs, the moDCs were harvested and washed. These moDCs were then recultured at varying doses with $10^{5}$ allogeneic T cells in triplicate round-bottomed 96-microwell plates without additional cytokines. DC doses ranged from 3,000 to 300 cells per well, yielding DC:T cell ratios from $1: 30$ to $1: 300$. $\left[{ }^{3} \mathrm{H}\right] \mathrm{TdR}$ uptake by proliferating allogeneic T cells over the last 12 hours of a 4-5 day culture was measured as an index of DC immunogenicity. (A) The averaged triplicate values (mean \pm SEM) for $\left[{ }^{3} \mathrm{H}\right] \mathrm{TdR}$ incorporation by $\mathrm{T}$ cells stimulated in 4 independent experiments using samples derived from $\mathrm{CD} 32 \mathrm{a}_{131} \mathrm{HH}$ or -HR donors are depicted logarithmically $\left(\log _{2}\right)$ on the $y$-axis. (B) Experiments using samples from $C D 32 a_{131} R R$ donors were performed in parallel, with immobilized mouse or human IgG ligating Fc $\gamma$ Rs on the immature moDCs. The averaged triplicate values (mean \pm SEM) from 3 independent experiments are depicted logarithmically $\left(\log _{2}\right)$ on the $y$ axis. Differences between conditions in $\mathbf{A}$ and $\mathbf{B}$ were tested using a stratified (by DC:T cell ratios) permutation $t$ test.

ated by targeting CD32a alone $(P<0.001)$. Specifically ligating $\mathrm{CD} 32 \mathrm{~b}$ (inverted triangles) did not support moDC maturation, so the stimulatory activity was no different than that in untreated immature DCs (squares). This is further evidence of the opposing functions of CD32a and CD32b on immature moDCs. These results also indicate that $\mathrm{CD} 32 \mathrm{~b}$ can actively inhibit $\mathrm{CD} 32 \mathrm{a}-$ mediated DC activation and immunogenicity, even in the context of a very strong stimulus like alloantigen.

The HH/HR versus RR phenotypes also affect reactivity to alloantigens in the alloMLR. Ligating immobilized human IgG to Fcys on immature moDCs from CD32 $\mathrm{a}_{131} \mathrm{RR}$ individuals did not significantly enhance immunogenicity (Figure 6B, triangles) compared with untreated immature moDCs (Figure 6B, squares). Coculturing these samples with immobilized mouse IgG (Figure 6B, circles), however, led to a marked increase in allostimulation $(P<0.001)$. This again confirms the functional significance of the CD32a $\mathrm{a}_{131}$ polymorphism and exemplifies how differences in the avidity of CD32a for IgG can create a functional shift in the overall balance between activating and inhibitory Fc $\gamma$ Rs.

\section{Discussion}

Previous studies of CD32 expression and function on human DCs $(4,25,28)$ could not distinguish between activating CD32a and inhibitory CD32b. We believe this is the first distinct characterization of CD32a and CD32b expression, modulation, and function on circulating human DCs, their precursors, and cytokine-induced moDCs. Nearly all circulating conventional DCs and a major population of moDCs express both CD32a and CD32b, which have opposing effects on IgG-mediated maturation and function of DCs. Ligating complexed human IgG to CD32a matures and activates moDCs in proportion to the frequency of CD32a expression, without any apparent bystander effect. This supports potent DC function in the alloMLR, a standard assay of DC immunogenicity, and in the release of proinflammatory cytokines. Ligation of CD32b significantly limits these immunogenic functions.

Cross-linking CD32a by complexed IgG induces phosphorylation of a cytoplasmic immunoreceptor tyrosine-based activation motif (ITAM) (29). In contrast, CD32b bears a cytoplasmic immunoreceptor tyrosine-based inhibition motif (ITIM). Coligation of $\mathrm{CD} 32 \mathrm{~b}$ initiates tyrosine phosphorylation of the ITIM that inhibits ITAM-mediated events (29). Coexpression of these divergent 
receptors, which share almost identical ligand-binding domains, therefore establishes a threshold of DC activation.

$\mathrm{CD} 32 \mathrm{~b}$ plays a major role in regulating immune responses in mice. Genetic deletion of $\mathrm{CD} 32 \mathrm{~b}$ predisposes to autoimmunity (30) and results in pathologically enhanced immune responses (31). We have studied the expression and modulation of CD32a and CD32b on the surface of live human cells with an antibody that detects an extracellular domain of CD32b. Unlike previously available reagents, this $\mathrm{mAb}$ does not cross-react with CD32a and finally permits an assessment of surface-expressed Fc $\gamma$ Rs. This is more physiologically relevant than prior methods that measured total $\mathrm{CD} 32 \mathrm{~b}$ protein or mRNA from cell lysates, which include the large intracellular compartment in addition to surface Fc $\gamma$ Rs.

Monocytes have emerged as the predominant effectors of ADCC against tumor cells in vivo (32). In contrast to their constitutive expression of the activating Fc $\gamma$ Rs, CD32a and CD64, monocyte expression of the inhibitory $\mathrm{CD} 32 \mathrm{~b}$ is surprisingly diverse, ranging from $1-48 \%$ among 30 healthy volunteers. This previously unrecognized variability in the balance between activating and inhibitory FcrRs in the resting state may influence the quality and/or magnitude of antibody-based immune responses.

Perturbations in the balance between activating and inhibitory Fc $\gamma$ Rs may affect the pathophysiology of autoimmune diseases (33) or the efficacy of antibody-based therapies $(8,34)$. For example, TNF- $\alpha$-mediated suppression of CD32a and CD32b may lead to decreased immune complex clearance in vivo. This may be one mechanism by which TNF- $\alpha$ contributes to an immune complexmediated disease like rheumatoid arthritis (35). While dexamethasone increases CD32a expression, it also increases CD32b and could shift the balance in favor of the inhibitory Fc $\gamma$ Rs, accounting for a treatment effect of steroids on autoimmune diseases. This may also underlie the negative effect of steroids on ADCC against tumors (36). Increased CD32b expression in other settings may actually improve therapeutic responses to IVIG (34). The addition of any adjunctive treatment to $\mathrm{mAb}$ therapy must therefore consider the consequences this has on therapeutic efficacy, owing to alterations in the ratio of activating to inhibitory FcyRs.

In a murine model of immune thrombocytopenic purpura, Samuelsson et al. showed that IVIG requires the presence of CD32b to mediate protective antiinflammatory effects and that IVIG increases CD32b expression on splenic macrophages (34). We found that antiinflammatory concentrations of soluble monomeric IgG decrease CD32a expression on immature moDCs without significantly affecting CD32b. Accordingly, soluble IgG-treated DCs do not gain allostimulatory capacity (data not shown) or increase production of inflammatory cytokines after coculture with immobilized IgG. The net effect on the balance between activating and inhibitory Fc $\gamma$ Rs on murine macrophages and human DCs is similar, however, and yields the same functional sequelae. Our findings are therefore consistent with those from mouse studies showing that the overall balance between activating and inhibitory $\mathrm{F} c \gamma \mathrm{Rs}$ is pertinent to the mode of action of IVIG (34).

Several lines of evidence show that optimum immune rejection of tumors or infectious pathogens requires coordinated cellular and humoral immune responses $(37,38)$. Though most often studied for their ability to stimulate antigen-specific T cells, DCs can also potently affect innate (39) and humoral (40) immune responses. We found that ligating CD32a on moDCs leads to secretion of IL-10 and IL-6, which stimulate B cells and plasma cells, and TNF- $\alpha$ and IL-8, which serve as chemoattractants for other innate effector cells like neutrophils. Despite the apparent Th2 type of response, and the absence of IL-12p70, CD32a-targeted DCs were able to stimulate more potent allogeneic $\mathrm{T}$ cell proliferation compared with control DCs. This reveals a unique type of maturation through which DCs can play a key role in recruiting a diversified immune response against antibody-coated pathogens or tumors.

Similar findings regarding the expression and function of CD32b on human moDCs were recently published (41). We did not find, however, that CD32b blockade increased secretion of IL-12p70. Our results are consistent with previous reports of absent or diminished IL-12p70 production after triggering CD32a on human monocytes $(42)$ and moDCs $(25,43)$. This discrepancy may derive from differences in methodology, e.g., the specific conditions of $\mathrm{Fc} \gamma \mathrm{R}$ blockade or subsequent removal versus inclusion of excess blocking $\mathrm{mAb}$ from DC cultures. We also used immobilized IgG to ligate the unblocked activating Fc $\gamma$ Rs, instead of relying on circulating immune complexes in serum that were diluted 100 -fold in culture (41). This may have led to differences in the extent of Fc $\gamma \mathrm{R}$ cross-linking and hence the divergent biological responses. We also blocked CD32b and recultured moDCs in the presence of $1 \%$ serum without immobilized IgG, and we did not find a statistically significant increase in moDC maturation or activation. We do not know whether blockade of CD32a in the other system would have prevented maturation and IL12p70 production or whether an alternative activation pathway might have been operative.

Blockade of cell activation has unique implications for DCs. While mature DCs are the most potent stimulators of immunity, immature DCs can mediate the opposite task of generating antigen-specific tolerance (9). We have shown that engaging CD32b, by first blocking CD32a or pretreating DCs with soluble IgG, limits IgG-mediated maturation and activation of moDCs, even in the context of a very strong immunogen such as alloantigen. CD32b helps maintain B cell tolerance (3), but we believe this is the first evidence that CD32b may play an active role in limiting DC maturation and hence in inducing $\mathrm{T}$ cell tolerance as well. That $\mathrm{CD} 32 \mathrm{~b}$ can mediate the uptake of immune complexes (44) suggests a rationale for targeting antigens to this receptor to induce antigenspecific tolerance, and such studies are underway. It also implies that tumor cells coated with therapeutic mAbs could inadvertently initiate an inhibitory pathway with undesirable results.

In the setting of fixed numbers of CD32a and CD32b surface epitopes, differences in functional avidity for IgG can shift their functional balance. In our studies using human IgG as ligand, the maturation and functional sequelae are most evident in samples from donors displaying the high-binding CD32 $\mathrm{a}_{131}$ alleles. Samples with low-affinity receptors are less affected by immobilized human IgG but are instead matured and activated by mouse IgG1. This indicates one of the mechanisms by which CD32a polymorphisms may affect immune responses to self or pathogenic/tumor antigens $(27,45)$. It also highlights how the species of IgG ligand can shape laboratory (6) or clinical studies (46) involving human Fc $\gamma$ Rs.

These data have shown that subsets of human DCs express a pair of receptors that share a common ligand but mediate opposing functions. These can elicit divergent immune responses. The balance of available activating versus inhibitory Fc $\gamma$ Rs determines the net response. We have altered this balance by using blocking $\mathrm{mAbs}$, modulating the expression of activating and/or inhibitory Fc $\gamma$ Rs, or using ligands of differing affinities for the respective Fc $\gamma$ Rs. These findings have important implications for elucidating the 
pathophysiology of autoimmune diseases, optimizing mAb therapies, engineering mAbs to target specific FcyRs (47), and rationally targeting Ags to FcyRs on DCs in vitro or in vivo.

\section{Methods}

Media, sera, and Igs. Complete RPMI 1640 was supplemented with $10 \mathrm{mM}$ HEPES, $1 \%$ penicillin/streptomycin (Media Laboratory, Memorial SloanKettering Cancer Center [MSKCC]), $50 \mu \mathrm{M}$ 2-ME (Invitrogen Corp.), 1\% L-glutamine (Invitrogen Corp.), and heat-inactivated NHS (Gemini BioProducts). All media and reagents were endotoxin free. Stock solution of therapeutic-grade sterile 10\% pooled human IgG (Gamunex; gift of Bayer HealthCare, Biological Products Division) was 100\% monomeric by fast protein liquid chromatography (data not shown). Pooled human IgG, mouse IgG1 (Sigma-Aldrich), and mouse IgG Fabs (Jackson ImmunoResearch Laboratories Inc.) were diluted immediately before use in sterile PBS.

Cytokines. Sterile recombinant, endotoxin-, pyrogen-, carrier-, and mycoplasma-free human cytokines used to support generation of moDCs in vitro and/or to modulate Fc $\gamma$ R expression included GM-CSF (Berlex); IL-1 $\beta$, IL-2, IL-3, IL-4, IL-6, IL-10, TGF- $\beta 1$, TNF- $\alpha$ (all from R\&D Systems); IFN- $\alpha 2 b$ (INTRON A; Schering Corp.); IFN- $\gamma-1 b$ (Actimmune; InterMune); and $\mathrm{PGE}_{2}$ (Calbiochem).

Cell purification and generation of DCs. Informed consent was obtained for research specimen collection using protocols approved by the Institutional Review and Privacy Board of Memorial Hospital, MSKCC. PBMCs were obtained from whole blood or leukocyte concentrates (Blood Bank, MSKCC) by density centrifugation over Ficoll-Paque PLUS (endotoxinfree, no. 17-1440-03; Amersham Pharmacia Biotech). Neutrophils were purified from the rbc pellet after hypotonic lysis of erythrocytes.

Circulating blood DCs were enriched from buffy coats by negative immunomagnetic selection according to the manufacturer's instructions (StemSep Human Dendritic Cell Enrichment Cocktail; StemCell Technologies). Enriched populations were cultured in 10\% NHS with IL-3 $(10 \mathrm{ng} / \mathrm{ml})$ to support CD11c-CD123 $3^{\text {bright }}$ plasmacytoid DCs (24) or without IL-3 for $\mathrm{CD} 11 \mathrm{c}^{+} \mathrm{CD} 123^{\text {low }}$ "myeloid" or conventional DCs. Immature moDCs were generated over 5-6 days from tissue culture plastic-adherent PBMCs (CD14 ${ }^{+}$monocyte precursors) in the presence of GM-CSF $(1000 \mathrm{IU} / \mathrm{ml})$ and IL-4 (500 IU/ml) in complete RPMI-1\% NHS (48). Fresh medium and cytokines were replenished every 2 days.

Antibodies and secondary reagents for staining and blocking. Direct FITC-, PE-, allophycocyanin-, PE-cyanine-5- (PC-5-), and PC-7-conjugated mouse anti-human mAbs included anti-CD3, anti-CD11c, anti-CD14, anti-CD16, anti-CD19, anti-CD20, anti-CD56, anti-CD64, anti-CD83(-FITC), antiCD86, anti-CD123, anti-HLA-DR (BD Biosciences - Pharmingen); and anti-CD83(-PE) (Immunotech). Anti-human CD32 mAbs included clone 41H16 (kind gift of Jan G.J. van de Winkel, University Medical Center, Utrecht, The Netherlands); clone IV.3 (HB-217; ATCC), with Fab fragments produced by the MSKCC Monoclonal Antibody Core Facility; clone 2B6 [described below; unconjugated and FITC-conjugated mouse IgG1 and FITC-conjugated chimeric $\mathrm{F}\left(\mathrm{ab}^{\prime}\right)_{2}$; MacroGenics]; PE-conjugated clone CIKm5 (CALTAG Laboratories); and FITC-conjugated clones FL18.26 and 3D3 (BD Biosciences - Pharmingen). Isotype controls included the appropriate fluorochrome-conjugated or unconjugated mouse IgG1, IgG2a, or IgG2b (DakoCytomation and BD Biosciences - Pharmingen). Unconjugated primary Igs were secondarily stained with FITC- or PE-conjugated $\mathrm{F}\left(\mathrm{ab}^{\prime}\right)_{2}$ fragments of goat anti-mouse IgG (Jackson ImmunoResearch Laboratories Inc.). Flow cytometry studies used a Cytomics FC 500 (Beckman Coulter), gating for collection and analysis of at least 10,000 live cells.

Production of $2 B 6 \mathrm{mAb}$ against $\mathrm{CD} 32 \mathrm{~b}$. Human CD32a-transgenic mice were immunized with a recombinant protein containing the complete extracellular domain of human CD32b. Spleen cells from immunized mice were fused with a proprietary myeloma cell line (MacroGenics Inc.). Resulting $\mathrm{mAbs}$ secreted by the hybridomas were screened by ELISA for differential binding of the inhibitory and activating isoforms of CD32. Specificity and selectivity were further confirmed by surface plasmon resonance analysis and flow cytometry of stained Burkitt lymphoma-derived cell lines and human peripheral blood leukocytes. 2B6 was a CD32b-blocking clone selected among several anti-CD32b-secreting hybridomas for its ability to react with $\mathrm{CD} 32 \mathrm{~b}$ at high titers with no binding to CD32a. Furthermore, clone $2 \mathrm{~B} 6$ blocked binding of aggregated human IgG to CD32b (M.-C. Veri et al., unpublished data).

Modulation of $F c \gamma R$ s. The following specific factors were added to immature moDC cultures starting on day 3 or 4 : IL-1 $\beta$ ( $2 \mathrm{ng} / \mathrm{ml})$, IL-2 $(6,000$ $\mathrm{IU} / \mathrm{ml}), \mathrm{IL}-3$ (10 ng/ml), IL-6 (1,000 IU/ml), IL-10 (25 ng/ml), IFN- $\gamma(1,000$ $\mathrm{U} / \mathrm{ml}), \mathrm{IFN}-\alpha 2 \mathrm{~b}(1,000 \mathrm{U} / \mathrm{ml}), \mathrm{PGE}_{2}(5 \mu \mathrm{M})$, TGF- $\beta$ (10 ng/ml), and TNF- $\alpha$ $(5 \mathrm{ng} / \mathrm{ml})$. IFN- $\gamma$-treated DCs were very adherent and required $5 \mathrm{~min}$ utes incubation with $0.4 \mu \mathrm{M}$ EDTA for harvesting. Pooled human IgG was added to yield a final concentration approaching that achieved after therapeutic administration of IVIG $(0.15 \mathrm{mM})$. Sterile therapeutic grade dexamethasone (American Regent Laboratories) was added to yield a final concentration of $0.1 \mu \mathrm{M}$. All conditions used only $1 \%$ NHS, except the one condition in which FCS was evaluated. Cells were harvested for phenotypic or functional studies on day 6-7.

To assess the effects of different maturation stimuli on Fc $\gamma$ R expression, the following were added to separate cultures of immature moDCs on day 6-7: cocktail of inflammatory cytokines (IL-1 $\beta$ [ $2 \mathrm{ng} / \mathrm{ml}]$, IL-6 $[1,000 \mathrm{IU} / \mathrm{ml}]$, TNF- $\alpha[10 \mathrm{ng} / \mathrm{ml}])$ and PGE $2(5 \mu \mathrm{M})(23), \mathrm{LPS}(10 \mathrm{ng} / \mathrm{ml})$, or CD40L-transfected murine fibroblasts (5 DCs per 1 CD40L-expressing fibroblast; kind gift of Jacques Banchereau, Baylor Institute for Immunology Research, Dallas, Texas, USA) (49). Cells were harvested 2 days later and evaluated for effects on Fc $\gamma$ R expression. Maturation was confirmed by upregulation of cell-surface CD83 on more than $90 \%$ of cells. Gating on HLA-DR ${ }^{\text {bright }}$ cells during cytofluorographic analysis excluded CD40L-transfected fibroblasts.

Quantitative flow cytometry. The frequencies of respective $\mathrm{Fc}_{\mathrm{R}} \mathrm{R}^{+}$cells per population, and relative changes thereof, were calculated as the number of viable immature (CD14-CD83-HLA-DR ${ }^{+}$) DCs with more intense staining than that of the isotype control, divided by the number of viable moDCs in the analysis gate. To quantify receptor density per cell, we used standardized antibody-binding beads (Quantum Simply Cellular; Bangs Laboratories Inc.), which comprise 4 populations with varying capacities to bind mouse IgG (e.g., detection mAbs) and 1 nonbinding blank population. The beads were stained and evaluated in parallel with moDCs using the same anti-Fc $\gamma$ R detection reagents. A standard calibration curve was generated by plotting the known antibody-binding capacity of each bead population against the measured MFI of the bound detection $\mathrm{mAb}$. We then calculated the number of detection $\mathrm{mAbs}$ bound per cell from the MFI of each sample using the standard curve. Whole IgG1 formulations of clones CIKm5 (antiCD32a) and 2B6 (anti-CD32b) were used at saturating concentrations to quantify CD32a and CD32b. Staining with Fab fragments of clone IV.3 and $\mathrm{F}\left(\mathrm{ab} \mathrm{b}_{2}\right)_{2}$ fragments of $2 \mathrm{~B} 6$ yielded the same changes in the frequency of CD32 $\mathrm{a}^{+}$or $\mathrm{CD} 32 \mathrm{~b}^{+}$DCs and shifts in MFI.

Ligation of Fcy Rs with immobilized IgG. We immobilized IgG on plastic plates to mimic the effect of complexed IgG (25). Pooled human or mouse IgG (both $1.0 \mathrm{mg} / \mathrm{ml}$ ) was added to each well of a 96-well round-bottom tissue culturetreated plate. Plates were washed 4 times with PBS after overnight incubation to yield immobilized IgG. For selective ligation of CD32a, harvested day 5-6 immature moDCs were washed with PBS; blocked with anti-CD32b (clone $2 \mathrm{~B} 6,5 \mu \mathrm{g} / \mathrm{ml}$ ) by incubation at $4^{\circ} \mathrm{C}$ for 30 minutes; washed with PBS 3 times to remove excess blocking $\mathrm{mAb}$; resuspended in complete medium supplemented with $1 \%$ NHS, GM-CSF, and IL-4; and recultured in 96-well plates 
with immobilized IgG ( $10^{5}$ cells $/ 0.1 \mathrm{ml} /$ well $)$. For selective ligation of CD $32 \mathrm{~b}$, cells were blocked with $\mathrm{mAb}$ IV.3 $(10 \mu \mathrm{g} / \mathrm{ml})$ before reculture with or without immobilized IgG. Preincubation without mAbs allowed simultaneous ligation of CD32a and CD32b. For each condition, cells added to plates lacking immobilized IgG served as negative controls. For experiments that used immobilized mouse IgG1, plates with immobilized Fab fragments of mouse IgG served as negative controls to rule out an effect of xenogeneic protein. Where indicated, treated moDCs were washed to remove excess soluble IgG or IFN- $\gamma$ before reculture with or without immobilized IgG.

T lymphocytes. $\mathrm{T}$ cells for alloMLRs were obtained from tissue culture plastic-nonadherent PBMCs, then further purified by nonadherence and elution from nylon wool columns (Polysciences Inc.). Purity was greater than $90-95 \%$ based on CD3 expression.

Cytokine measurement by cytometric bead array. Supernatants of DCs were collected 2 days after $\mathrm{F} \gamma \mathrm{R}$ ligation, immediately frozen, and thawed once for assay. Levels of IL-1 $\beta$, IL-6, IL-8, IL-10, IL-12p70, and TNF- $\alpha$ were measured simultaneously using a Human Inflammation Cytometric Bead Array (CBA) Kit (BD Biosciences - Pharmingen) according to the manufacturer's instructions.

AlloMLRs. DCs were harvested 48 hours after Fc $\gamma \mathrm{R}$ ligation, extensively washed, then cocultured with $10^{5}$ purified allogeneic T cells in triplicate round-bottomed microwells (Costar 3799; Corning Inc.) at variable ratios from 1:30 to 1:300 (DC:T cells), in complete RPMI 1640 supplemented with $10 \%$ NHS. Methyl- $\left[{ }^{3} \mathrm{H}\right]$ thymidine $\left(\left[{ }^{3} \mathrm{H}\right] \mathrm{TdR} ; 1 \mu \mathrm{Ci} /\right.$ well; New England Nuclear, PerkinElmer) was added to each well on day 4-5, and plates were harvested 10-12 hours later using a Harvester 96 Mach III (Tomtec). Proliferation of responder $\mathrm{T}$ cells was based on $\left[{ }^{3} \mathrm{H}\right] \mathrm{TdR}$ incorporation, which was measured using a microplate scintillation counter (TopCount NXT; PerkinElmer).

Biostatistics. The 2-sample $t$ test was used to compare expression of CD83 and CD86, and concentrations of IL-10, IL- 6 , and TNF- $\alpha$ between experi- mental conditions. Due to truncation of values at 5,000, the Mann-Whitney $U$ test was used to compare concentrations of IL-8. We analyzed differences in $\left[{ }^{3} \mathrm{H}\right] \mathrm{TdR}$ uptake between conditions in the alloMLR across a range of DC:T cell ratios. The differences were tested using a stratified (by DC:T cell ratios) permutation $t$ test, where the subject was the permutation unit, in order to maintain the correlation structure between $\mathrm{T}$ cell doses. $P$ values less than or equal to 0.05 were considered statistically significant.

\section{Acknowledgments}

This work was supported by grants from the Charles A. Dana Foundation and the Mortimer J. Lacher Fellowship Fund, MSKCC (to A.M. Boruchov); the National Cancer Institute, NIH (R01 CA 83070 to J.W. Young; P01 CA 59350 to J.W. Young and G. Heller; and P01 CA 23766 to J.W. Young and G. Heller); the National Institute of Allergy and Infectious Diseases, NIH (P01 AI 51573 to J.V. Ravetch); the Alliance for Lupus Research (to J.V. Ravetch); and William H. Goodwin and Alice Goodwin of the Commonwealth Foundation for Cancer Research through The Experimental Therapeutics Center of MSKCC (to A.M. Boruchov and J.W. Young). We appreciate the technical assistance of Michael Basedow.

Received for publication February 15, 2005, and accepted in revised form June 28, 2005.

Address correspondence to: Adam M. Boruchov or James W. Young, Memorial Sloan-Kettering Cancer Center, 1275 York Avenue, New York, New York 10021, USA. Phone: (212) 6397169; Fax: (212) 717-3588; E-mail: boruchoa@mskcc.org (A.M. Boruchov). Phone: (212) 639-7989; Fax: (212) 717-3371; E-mail: youngjw@mskcc.org (J.W. Young).
1. Waldmann, T.A. 2003. Immunotherapy: past, present and future. Nat. Med. 9:269-277.

2. Edwards, J.C.W., et al. 2004. Efficacy of B-cell-targeted therapy with rituximab in patients with rheumatoid arthritis. N. Engl. J. Med. 350:2572-2581.

3. Ravetch, J.V., and Bolland, S. 2001. IgG Fc receptors. Annu. Rev. Immunol. 19:275-290.

4. Fanger, N.A., Wardwell, K., Shen, L., Tedder, T.F., and Guyre, P.M. 1996. Type 1 [CD64] and type 11 [CD32] Fc gamma receptor-mediated phagocytosis by human blood dendritic cells. J. Immunol. 157:541-548

5. Regnault, A., et al. 1999. Fcgamma receptor-mediated induction of dendritic cell maturation and major histocompatibility complex class I-restricted antigen presentation after immune complex internalization. J. Exp. Med. 189:371-380.

6. Dhodapkar, K.M., Krasovsky, J., Williamson, B., and Dhodapkar, M.V. 2002. Antitumor monoclonal antibodies enhance cross-presentation of cellular antigens and the generation of myelomaspecific killer T cells by dendritic cells. J. Exp. Med. 195:125-133.

7. Kalergis, A.M., and Ravetch, J.V. 2002. Inducing tumor immunity through the selective engagement of activating Fcgamma receptors on dendritic cells. J. Exp. Med. 195:1653-1659.

8. Clynes, R.A., Towers, T.L., Presta, L.G., and Ravetch, J.V. 2000. Inhibitory Fc receptors modulate in vivo cytoxicity against tumor targets. Nat. Med. 6:443-446.

9. Mellman, I., and Steinman, R.M. 2001. Dendritic cells: specialized and regulated antigen processing machines [review]. Cell. 106:255-258.

10. Van Sorge, N.M., Van der Pol, W.L., and Van de Winkel, J.G. 2003. FcgammaR polymorphisms: implications for function, disease susceptibility and immunotherapy [review]. Tissue Antigens.
61:189-202.

11. Schlossman, S.F., et al. 1995. Leucocyte typing $V$ : white cell differentiation antigens. Oxford University Press. Oxford, United Kingdom/New York, New York, USA. 2044 pp.

12. Gamberale, R., et al. 2003. Expression of Fcgamma receptors type II (FcgammaRII) in chronic lymphocytic leukemia B cells. Blood. 102:2698-2699.

13. Maresco, D.L., Osborne, J.M., Cooney, D., Coggeshall, K.M., and Anderson, C.L. 1999. The SH2-containing $5^{\prime}$-inositol phosphatase (SHIP) is tyrosine phosphorylated after Fcgamma receptor clustering in monocytes. J. Immunol. 162:6458-6465.

14. Parren, P.W., et al. 1992. On the interaction of IgG subclasses with the low affinity Fc gamma RIIa (CD32) on human monocytes, neutrophils, and platelets. Analysis of a functional polymorphism to human IgG2. J. Clin. Invest. 90:1537-1546.

15. Denomme, G.A., et al. 1997. Activation of platelets by sera containing IgG1 heparin-dependent antibodies: an explanation for the predominance of the Fc gammaRIIa "low responder" (his131) gene in patients with heparin-induced thrombocytopenia. J. Lab. Clin. Med. 130:278-284.

16. Vely, F., et al. 1997. A new set of monoclonal antibodies against human Fc gamma RII (CD32) and Fc gamma RIII (CD16): characterization and use in various assays. Hybridoma. 16:519-528.

17. Warmerdam, P., van de Winkel, J., Gosselin, E., and Capel, P. 1990. Molecular basis for a polymorphism of human Fc gamma receptor II (CD32). J. Exp. Med. 172:19-25.

18. Looney, R.J., Anderson, C.L., Ryan, D.H., and Rosenfeld, S.I. 1988. Structural polymorphism of the human platelet Fc gamma receptor. J. Immunol. 141:2680-2683.

19. Siegal, F.P., et al. 1999. The nature of the principal type 1 interferon-producing cells in human blood.
Science. 284:1835-1837.

20. Dzionek, A., et al. 2000. BDCA-2, BDCA-3, and BDCA-4: three markers for distinct subsets of dendritic cells in human peripheral blood. J. Immunol. 165:6037-6046.

21. Passlick, B., Flieger, D., and Ziegler-Heitbrock, H.W. 1989. Identification and characterization of a novel monocyte subpopulation in human peripheral blood. Blood. 74:2527-2534.

22. Geissmann, F., Jung, S., and Littman, D.R. 2003. Blood monocytes consist of two principal subsets with distinct migratory properties. Immunity. 19:71-82.

23. Jonuleit, H., et al. 1997. Pro-inflammatory cytokines and prostaglandins induce maturation of potent immunostimulatory dendritic cells under fetal calf serum-free conditions. Eur. J. Immunol. 27:3135-3142.

24. Grouard, G., et al. 1997. The enigmatic plasmacytoid T cells develop into dendritic cells with IL-3 and CD40-ligand. J. Exp. Med. 185:1101-1111.

25. Banki, Z., et al. 2003. Cross-linking of CD32 induces maturation of human monocyte-derived dendritic cells via NF-kappaB signaling pathway. J. Immunol. 170:3963-3970.

26. Zhou, L.-J., and Tedder, T.F. 1995. Human blood dendritic cells selectively express CD83, a member of the immunoglobulin superfamily. J. Immunol. 154:3821-3835.

27. Weng, W.K., and Levy, R. 2003. Two immunoglobulin $\mathrm{G}$ fragment $\mathrm{C}$ receptor polymorphisms independently predict response to rituximab in patients with follicular lymphoma. J. Clin. Oncol. 21:3940-3947.

28. Sallusto, F., and Lanzavecchia, A. 1994. Efficient presentation of soluble antigen by cultured human dendritic cells is maintained by granulocyte/macrophage colony-stimulating factor plus interleukin 4 
and downregulated by tumor necrosis factor $\alpha$. J. Exp. Med. 179:1109-1118.

29. Ravetch, J.V., and Lanier, L.L. 2000. Immune inhibitory receptors. Science. 290:84-89.

30. Bolland, S., and Ravetch, J.V. 2000. Spontaneous autoimmune disease in $\mathrm{Fc}$ (gamma)RIIB-deficient mice results from strain-specific epistasis. Immunity. 13:277-285.

31. Clatworthy, M.R., and Smith, K.G. 2004. FcgammaRIIb balances efficient pathogen clearance and the cytokine-mediated consequences of sepsis. J. Exp. Med. 199:717-723.

32. Uchida, J., et al. 2004. The innate mononuclear phagocyte network depletes B lymphocytes through $F c$ receptor-dependent mechanisms during anti-CD20 antibody immunotherapy. J. Exp. Med. 199:1659-1669.

33. McGaha, T.L., Sorrentino, B., and Ravetch, J.V. 2005. Restoration of tolerance in lupus by targeted inhibitory receptor expression. Science. 307:590-593.

34. Samuelsson, A., Towers, T.L., and Ravetch, J.V. 2001. Anti-inflammatory activity of IVIG mediated through the inhibitory Fc receptor. Science. 291:484-486.

35. Charles, P., et al. 1999. Regulation of cytokines, cytokine inhibitors, and acute-phase proteins following anti-TNF-alpha therapy in rheumatoid arthritis. J. Immunol. 163:1521-1528.
36. Rose, A.L., Smith, B.E., and Maloney, D.G. 2002. Glucocorticoids and rituximab in vitro: synergistic direct antiproliferative and apoptotic effects. Blood. 100:1765-1773.

37. Dyall, R., Vasovic, L.V., Clynes, R.A., and NikolicZugic, J. 1999. Cellular requirements for the monoclonal antibody-mediated eradication of an established solid tumor. Eur. J. Immunol. 29:30-37.

38. Amigorena, S. 2002. Fc gamma receptors and cross-presentation in dendritic cells. J. Exp. Med. 195:F1-F3.

39. Munz, C., et al. 2005. Mature myeloid dendritic cell subsets have distinct roles for activation and viability of circulating human natural killer cells. Blood. 105:266-273.

40. Dubois, B., et al. 1997. Dendritic cells enhance growth and differentiation of CD40-activated B lymphocytes. J. Exp. Med. 185:941-951.

41. Dhodapkar, K.M., et al. 2005. Selective blockade of inhibitory Fcgamma receptor enables human dendritic cell maturation with IL-12p70 production and immunity to antibody-coated tumor cells. Proc. Natl. Acad. Sci. U. S. A. 102:2910-2915.

42. Berger, S., Chandra, R., Ballo, H., Hildenbrand, R., and Stutte, H.J. 1997. Immune complexes are potent inhibitors of interleukin-12 secretion by human monocytes. Eur. J. Immunol. 27:2994-3000.

43. Radstake, T.R., et al. 2004. High production of proinflammatory and Th 1 cytokines by dendritic cells from patients with rheumatoid arthritis, and down regulation upon FcgammaR triggering. Ann. Rheum. Dis. 63:696-702.

44. Amigorena, S., et al. 1992. Cytoplasmic domain heterogeneity and functions of IgG Fc receptors in B lymphocytes. Science. 256:1808-1812.

45. Carcao, M.D., et al. 2003. Fcgamma receptor IIa and IIIa polymorphisms in childhood immune thrombocytopenic purpura. Br. J. Haematol. 120:135-141.

46. Tax, W.J., Willems, H.W., Reekers, P.P., Capel, P.J., and Koene, R.A. 1983. Polymorphism in mitogenic effect of IgG1 monoclonal antibodies against T3 antigen on human T cells. Nature. 304:445-447.

47. Armour, K.L., van de Winkel, J.G., Williamson, L.M., and Clark, M.R. 2003. Differential binding to human FcgammaRIIa and FcgammaRIIb receptors by human IgG wildtype and mutant antibodies. Mol. Immunol. 40:585-593.

48. Thurner, B., et al. 1999. Generation of large numbers of fully mature and stable dendritic cells from leukapheresis products for clinical application [review]. J. Immunol. Methods. 223:1-15.

49. de Saint-Vis, B., et al. 1998. A novel lysosome-associated membrane glycoprotein, DC-LAMP, induced upon DC maturation, is transiently expressed in MHC class II compartment. Immunity. 9:325-336. 